\title{
Effect of particle beam radiotherapy on locally recurrent rectal cancer: Three case reports
}

\author{
YUKAKO MOKUTANI, HIROFUMI YAMAMOTO, MAMORU UEMURA, NAOTSUGU HARAGUCHI, \\ HIDEKAZU TAKAHASHI, JUNICHI NISHIMURA, TAISHI HATA, ICHIRO TAKEMASA, \\ TSUNEKAZU MIZUSHIMA, YUICHIRO DOKI and MASAKI MORI
}

Department of Gastroenterological Surgery, Graduate School of Medicine, Osaka University, Suita, Osaka 565-0871, Japan

Received February 9, 2015; Accepted February 17, 2015

DOI: $10.3892 / \mathrm{mco} .2015 .532$

\begin{abstract}
Surgical resection is the most effective therapy for locally recurrent rectal cancer (LRRC); however, it often necessitates invasive procedures that may lead to major complications. Particle beam radiotherapy (RT), including carbon ion RT (C-ion RT) and proton beam RT, is a promising new modality that exhibits considerable efficacy against various types of human cancer. C-ion RT reportedly offers a therapeutic alternative for LRRC. In the present study, we describe three cases of LRRC treated by particle beam RT. In all the cases, LRRC was diagnosed by computed tomography, magnetic resonance imaging and positron emission tomography imaging. No serious adverse effects were observed during RT. One patient experienced re-recurrence of LRRC, but survived for 6 years following particle beam RT; the second patient remains recurrence-free after a 2-year follow-up; and the third patient has developed recurrence at different sites in the pelvis but, to date, has survived for 4 years following particle beam RT. Therefore, LRRC was controlled by particle beam RT in two of the three cases, suggesting that particle beam RT is a safe alternative treatment for patients with LRRC.
\end{abstract}

\section{Introduction}

Rectal cancer is a widespread malignancy with a high incidence rate. Advances in surgical techniques have decreased the frequency of postoperative rectal cancer recurrence $(1,2)$, although the rate remains high. In Japan, the reported disease recurrence rates are $7.3 \%$ for hepatic metastasis, $7.5 \%$ for

Correspondence to: Dr Hirofumi Yamamoto, Department of Gastroenterological Surgery, Graduate School of Medicine, Osaka University, 2-2 Yamadaoka, Suita, Osaka 565-0871, Japan

E-mail: hyamamoto@gesurg.med.osaka-u.ac.jp

Abbreviations: LRRC, locally recurrent rectal cancer; C-ion RT, carbon ion radiotherapy; TPE, total pelvic exenteration; CT, computed tomography; LTE, linear energy transfer; NIRS, National Institute of Radiological Sciences

Key words: local recurrence, rectal cancer, particle beam radiotherapy, carbon ion radiotherapy, proton beam radiotherapy lung metastasis and $8.8 \%$ for locally recurrent rectal cancer (LRRC) (3).

Surgical resection is considered the most effective curative treatment for postoperative LRRC in the pelvis. However, resection is less commonly performed for LRRC compared to liver or lung metastases. A recent meta-analysis (4) concluded that the primary goal of LRRC treatment is to achieve radical resection. However, such surgical resection often requires a highly aggressive approach, such as total pelvic exenteration, and is associated with a high risk of postoperative complications, including massive hemorrhage and severe sepsis. Additionally, postoperative fibrosis and adhesions are often indistinguishable from the recurrent tumor, making curative resection even more difficult $(5,6)$.

Particle beams utilizing protons and carbon ions (C-ions) provide a more favorable dose distribution compared with those involving photons. Furthermore, C-ions are heavier compared to protons and, thus, have greater biological effectiveness. C-ion radiotherapy (RT) was first utilized in 1994 by the National Institute of Radiological Sciences. C-ion RT was tested on a variety of tumor types in clinical trials and was approved as a highly advanced medical technology by the Japanese Ministry of Health in 2003. The main types of cancer that have been treated with C-ion RT include bone and soft tissue sarcoma, prostate, head and neck and non-small-cell lung cancer. Particle beam RT, including C-ion RT, has also been investigated as a potential novel tool for controlling LRRC. This is the report of three cases of LRRC treated using particle beam RT.

\section{Case reports}

Patient selection criteria. Particle beam RT treatment for LRRC was indicated for patients who refused to undergo surgery and who were able to cover the high treatment cost ( $\sim$ three million yen). Additionally, the recurrent tumors should not be closely adjacent to the intestine or colon and rectum. Particle beam RT was performed at the Hyogo Ion Beam Medical Center, the first institute in Japan to provide both proton and $\mathrm{C}$-ion therapy.

Therapeutic planning. Computed tomography (CT) scans were performed for therapeutic planning. The specific therapy technique was as previously described (7-9). Briefly, the CT 
A
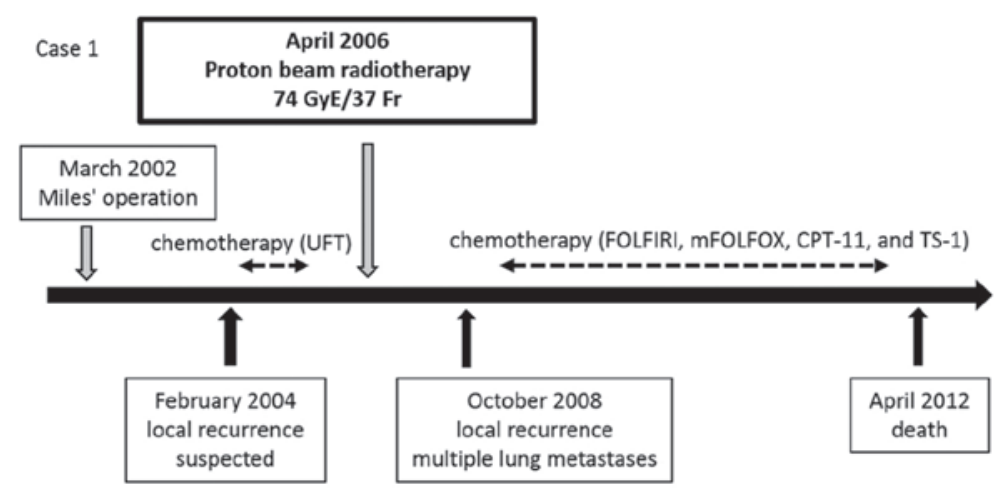

B

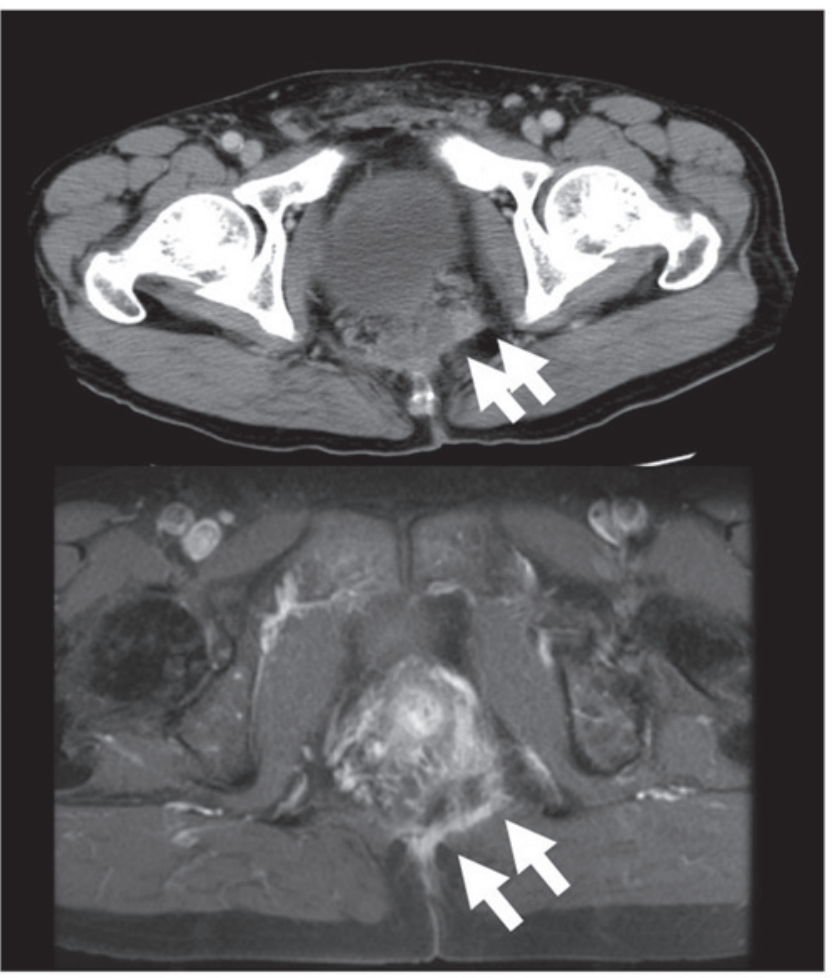

C

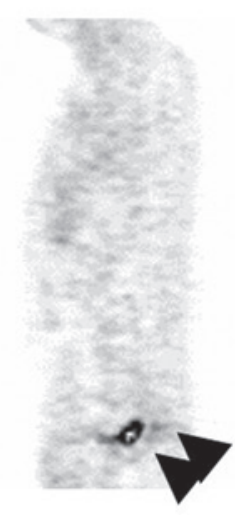

Figure 1. Summary of case 1. (A) Course of treatment following surgical resection. (B) Computed tomography (CT) image from February, 2004. (C) Positron emission tomography-CT scans from February, 2004 showing faint fluorodeoxyglucose uptake in soft tissues (arrowheads) in accordance with the CT images. (D) Magnetic resonance image from March, 2006 showing tumor invasion of the caudal space (arrows). UFT, tegafur-uracil; FOLFIRI, fluorouracil, leucovorin and irinotecan; mFOLFOX, modified leucovorin, fluorouracil and oxaliplatin; CPT-11, irinotecan; TS-1, tegafur, gimeracil and oteracil potassium.

imaging data were transferred to the treatment planning system. Irradiation parameters, in terms of the number of irradiation portals and their directions, were determined in conjunction with target volume delineation. A 5-mm safety margin was usually added to the clinical target volume to create the planning target volume. The dose in Gray equivalents $(\mathrm{GyE}=$ carbon physical dose in gray $\mathrm{x}$ relative biological effectiveness) was calculated for the target volume and any nearby critical structures, such as skin, urinary bladder and intestines.

Written informed consent was obtained from the patient or their family for publication of this manuscript and accompanying images.

Case 1. A 56-year-old man with lower rectal cancer and lymph node metastasis (stage IIIA according to the Union for International Cancer Control tumor-node-metastasis classification) underwent curative abdominoperineal excision
(Miles' operation) in March, 2002 (Fig. 1A). The histological evaluation established the diagnosis of moderately differentiated adenocarcinoma. At the patient's request, no postsurgical adjuvant chemotherapy was administered. In February, 2004, CT and positron emission tomography (PET) scans revealed LRRC in the patient's pelvis (Figs. 1B and C). Tegafur-uracil was orally administered for 2 years. In April, 2006, a magnetic resonance imaging scan showed that the LRRC lesion had increased in size (Fig. 1D) and the patient underwent proton beam RT. During RT, the patient exhibited only grade 1 dermatitis and he completed the entire course of treatment (74 GyE/37 Fr). In October, 2008, the patient exhibited multiple lung metastases and reappearance of the LRRC. In March, 2010, the LRRC invaded the sacrum and the patient was initiated on pain control medication due to sacral nerve invasion. The patient underwent a series of chemotherapy treatments, including FOLFIRI (fluorouracil, leucovorin and irinotecan), mFOLFOX (modified leucovorin, fluorouracil and 
A

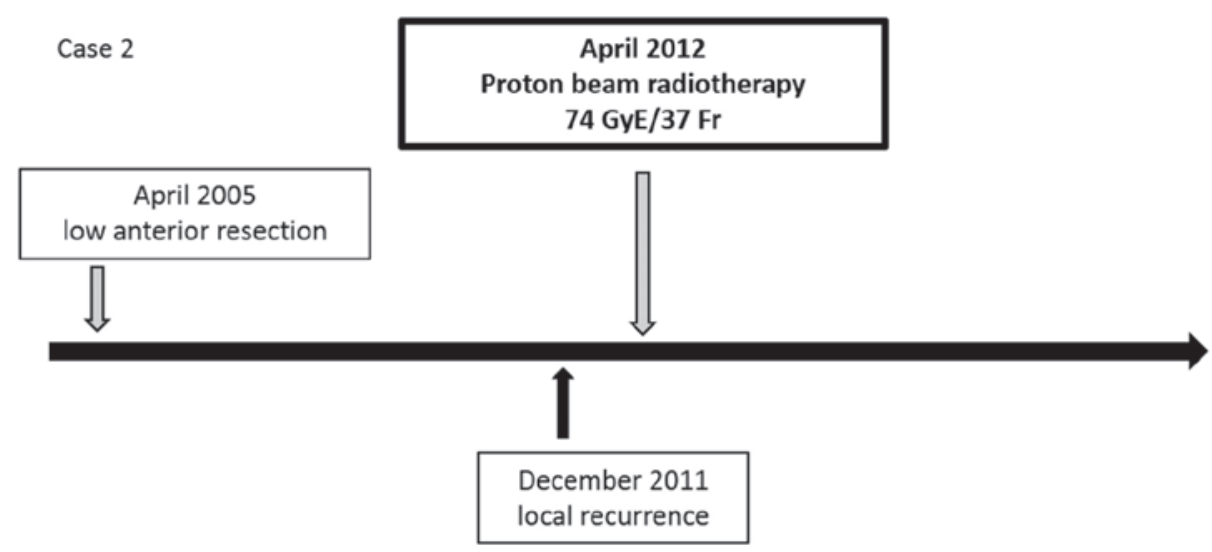

B
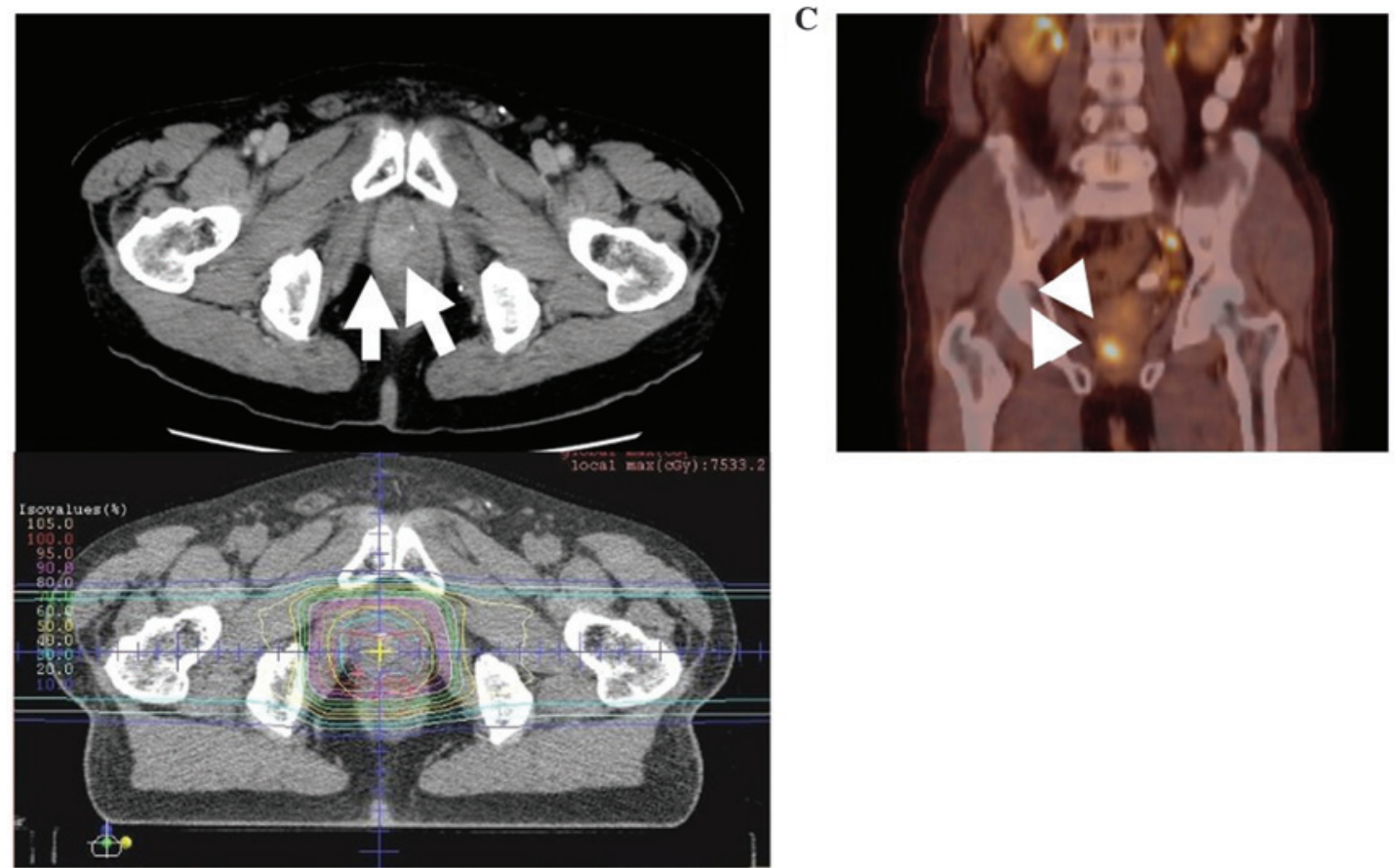

D

Figure 2. Summary of case 2. (A) Course of treatment following surgical resection. (B) Computed tomography (CT) image from December 2011 showing the locally recurrent rectal cancer near the right side of the prostate (arrows). (C) Positron emission tomography-CT from December 2011 showing fluorodeoxyglucose uptake (arrowheads) in accordance with the CT image. (D) Treatment planning for radiotherapy.

oxaliplatin), irinotecan and TS-1 (tegafur, gimeracil and oteracil potassium), but succumbed to the disease in April, 2012.

Case 2. In April, 2005, a 70-year-old man with lower rectal cancer and lymph node metastasis (stage IIIA) underwent low anterior resection with curative intent (Fig. 2A). The histological evaluation established the diagnosis of moderately differentiated adenocarcinoma. No postoperative adjuvant chemotherapy was administered due to the patient's advanced age and chronic heart disease. Six years after the surgery, in December, 2011, LRRC was detected by CT (Fig. 2B) and PET (Fig. 2C) scans. Proton beam RT was performed in April, 2012 (Fig. 2D). During RT, the patient experienced no adverse events and the treatment was completed ( $74 \mathrm{GyE} / 37 \mathrm{fr}$ ). The patient has remained tumor recurrence-free for $>2.5$ years.

Case 3. In September 2005, a 71-year-old man with lower rectal cancer underwent endoscopic mucosal resection (EMR)
(Fig. 3A). The histological evaluation revealed vessel invasion and deep invasion into the submucosal layer, necessitating additional surgical treatment. However, the patient firmly declined additional surgery; therefore, he underwent periodic CT scans, colonoscopy and blood tests to monitor disease recurrence or distant metastasis. Four years later, the patient exhibited rectal cancer recurrence on the EMR site. Intersphincteric resection was performed in May, 2009. The histological examination revealed that the tumor was a well-differentiated adenocarcinoma (stage I). In June, 2010, PET-CT scans revealed LRRC in the patient's pelvis and C-ion RT was performed in September, 2010. No adverse events were observed during the RT and the patient completed the treatment (70.4 GyE/16 fr). To date, there has been no LRRC; however, the patient has had disease recurrence at different sites in the pelvis. The patient remained symptom-free until December, 2014, but he currently requires medication with oral morphine for severe perianal pain. 
A
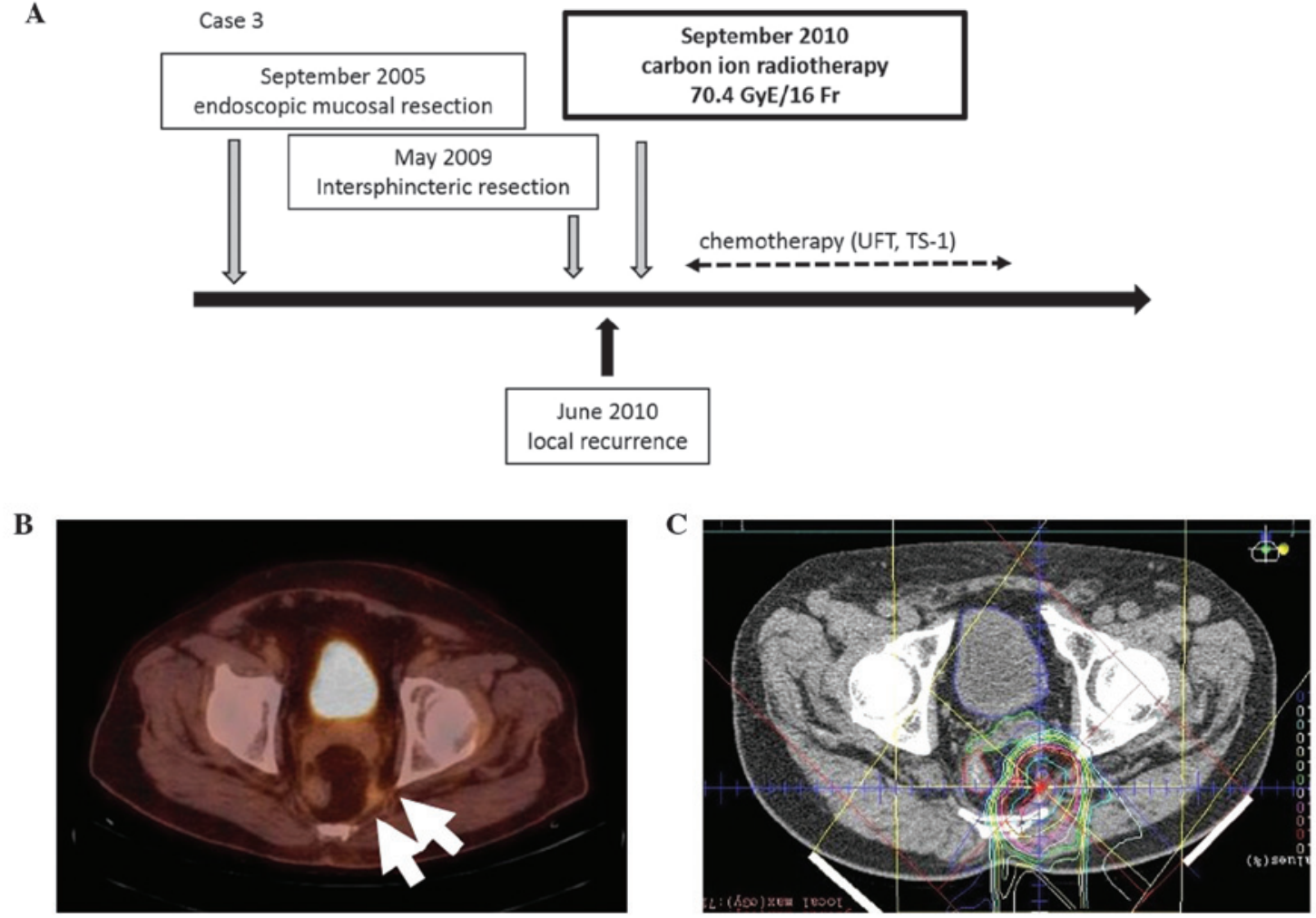

Figure 3. Summary of case 3. (A) Course of treatment following endoscopic mucosal resection. (B) Positron emission tomography-computed tomography image from June, 2010 showing locally recurrent rectal cancer in the pelvic floor (arrows). (C) Treatment planning for radiotherapy. UFT, tegafur-uracil; TS-1, tegafur, gimeracil and oteracil potassium.

\section{Discussion}

To date, surgical resection remains the standard therapy for LRRC, with continuous advances in the surgical techniques. However, the 5-year survival rate following surgical treatment for LRRC remains 17-36\% (5,6,10,11). Furthermore, surgical treatment often requires the creation of two stomas, which significantly compromises the patient's quality of life. Additionally, sacral nerve damage caused by high sacral resection may lead to walking disorders and severe lower limb pain. Particle beam RT may be a potentially useful alternative treatment option in certain cases of LRRC.

Particle beams possess several unique physical and biological properties, such as the sharp, narrow Bragg peak created by the energy deposition (high-dose peak) at the end of the beam path. In contrast with photons and fast neutrons, the peak of a particle beam is narrower, providing a dosage at the peak that is several times higher compared with the dose in the plateau. A flow of hydrogen nuclei is referred to as a proton beam, while a flow of carbon nuclei is called a C-ion beam. The particle range is controlled by the energy of the incoming particles. Photons, electrons and protons are referred to as low-linear energy transfer (LTE) radiations, while a C-ion is considered ionizing high-LTE radiation. High-LTE radiation may cause cellular damage, regardless of cell cycle phase and oxygenation status (8). The C-ion beam is a minimally invasive radiation technology that delivers a large dose of highly focused ionizing radiation to the target tumor, thus reducing toxicity to normal tissues.
The use of $\mathrm{C}$-ion $\mathrm{RT}$ to treat postoperative pelvic recurrence of rectal cancer has been previously reported. Yamada et al (12) performed a dose-escalation study and demonstrated that the local control and survival rates correlated to the total dose delivered. They reported 5-year survival rates of $24,27.5$ and $42.3 \%$ for patients treated with $67.2,70.4$ and $73.6 \mathrm{GyE} / 16 \mathrm{fr}$, respectively. Their study design did not restrict other treatments, including chemotherapy and molecularly targeted therapy; however, their results were compatible with the surgical outcomes. No adverse events were reported in the acute phase with $\mathrm{C}$-ion RT of $73.6 \mathrm{GyE} / 16 \mathrm{fr}$; therefore, the survival rate at this dose is acceptable as compared with that of surgical resection of LRRC.

In the present study, LRRC was treated using irradiation with proton beam RT of $74 \mathrm{GyE} / 37$ fr for cases 1 and 2 and with $\mathrm{C}$-ion RT of $70.4 \mathrm{GyE} / 16$ fr for case 3 . In each case, treatment was determined considering the tumor size and the patient's basal complications. In cases 2 and 3, treatment successfully led to long-term control of LRRC, with survival over a follow-up period of 2-4 years. The patient in case 1 eventually succumbed to the disease, but the local recurrence remained stable for 2 years following RT and he survived for 6 years after the treatment. None of the treated patients experienced severe adverse events during the therapy, although two patients were aged $>75$ years. Our results did not reveal significant differences between the proton beam RT and the $\mathrm{C}$-ion RT with regard to controlling LRRC. It is possible that sequential therapy and chemotherapy or surgery may improve the curative rate of LRRC. Tomokuni et al (13) previously 
reported a case of LRRC treated with curative surgery and preoperative $\mathrm{C}$-ion $\mathrm{RT}$.

In case 1 , the patient developed inflammation of the pelvic dead space following surgery, as well as long-lasting discharge from the perineal wound. LRRC was first suspected in February, 2004. However, we could not definitively differentiate between inflammatory changes and disease recurrence until the tumor marker carcinoembryonic antigen levels increased to $>100 \mathrm{ng} / \mathrm{ml}$ in 2005. Although there is no consensus regarding the optimal time to initiate RT, it is possible that earlier induction would have improved the prognosis in case 1 .

In conclusion, particle beam RT is an effective treatment for local control of LRRC in patients for whom surgical resection is not considered the optimal choice. Particle beam RT represents a promising alternative to surgery. However, the long-term safety of this approach requires further investigation.

\section{References}

1. Dias AR and Nahas SC: Modified supralevator pelvic exenteration for the treatment of locally advanced rectal cancer with vaginal and uterine invasion. Surg Today 43: 702-704, 2013.

2. Seishima R, Okabayashi K, Hasegawa H, Sugiyama D, Ishii Y, Tsuruta M, Takebayashi T and Kitagawa Y: Obesity was associated with a decreased postoperative recurrence of rectal cancer in a Japanese population. Surg Today 44: 2324-2331, 2014.

3. Watanabe T, Itabashi M, Shimada Y, et al; Japanese Society for Cancer of the Colon and Rectum: Japanese Society for Cancer of the Colon and Rectum (JSCCR) guidelines 2010 for the treatment of colorectal cancer. Int J Clin Oncol 17: 1-29, 2012.
4. Bhangu A, Ali SM, Darzi A, Brown G and Tekkis P: Meta-analysis of survival based on resection margin status following surgery for recurrent rectal cancer. Colorectal Dis 14: 1457-1466, 2012.

5. Ike H, Shimada H, Ohki S, Yamaguchi S, Ichikawa Y and Fujii S: Outcome of total pelvic exenteration for locally recurrent rectal cancer. Hepatogastroenterology 50: 700-703, 2003.

6. Nielsen MB, Rasmussen PC, Lindegaard JC and Laurberg S: A 10-year experience of total pelvic exenteration for primary advanced and locally recurrent rectal cancer based on a prospective database. Colorectal Dis 14: 1076-1083, 2012.

7. Kamada T: Clinical evidence of particle beam therapy (carbon). Int J Clin Oncol 17: 85-88, 2012.

8. Tsujii $\mathrm{H}$ and Kamada T: A review of update clinical results of carbon ion radiotherapy. Jpn J Clin Oncol 42: 670-685, 2012.

9. Okada T, Kamada T, Tsuji H, et al: Carbon ion radiotherapy: Clinical experiences at National Institute of Radiological Science (NIRS). J Radiat Res (Tokyo) 51: 355-364, 2010.

10. Bosman SJ, Vermeer TA, Dudink RL, de Hingh IH, Nieuwenhuijzen GA and Rutten HJ: Abdominosacral resection: Long-term outcome in 86 patients with locally advanced or locally recurrent rectal cancer. Eur J Surg Oncol 40: 699-705, 2014.

11. Moriya Y, Akasu T, Fujita S and Yamamoto S: Total pelvic exenteration with distal sacrectomy for fixed recurrent rectal cancer in the pelvis. Dis Colon Rectum 47: 2047-2053; discussion 2053-4, 2004.

12. Yamada S, Shinoto M and Endo S: Carbon ion radiotherapy for patients with locally recurrent rectal cancer. Proceedings of NIRS-ETOILE 2nd Joint Symposium on Carbon Ion Radiotherapy 2011. NIRS-M-243: 54-59, 2011.

13. Tomokuni A, Takahashi H, Ikeda M, Mizushima T, Takemasa I, Yamamoto H, Sekimoto M, Yamada S, Doki Y and Mori M: A case of locally recurrent rectal cancer resected in combination with preoperative carbon ion radiotherapy. Jpn J Gastroenterol Surg 43: 595-600, 2010. 